\title{
Análise da técnica de pedalada durante o ciclismo até a exaustão
}

\author{
Fernando Diefenthaeler ${ }^{1}$ \\ Rodrigo Rico Bini ${ }^{2}$ \\ Marco Aurélio Vaz ${ }^{3}$ \\ ${ }^{1}$ Laboratório de Biomecânica, Universidade Federal de Santa Catarina, Florianópolis, SC, Brasil \\ ${ }^{2}$ Sports Performance Research Institute, School of Sport and Recreation, Auckland University of \\ Technology, North Shore City, New Zealand \\ ${ }^{3}$ Laboratório de Pesquisa do Exercício, Universidade Federal do Rio Grande do Sul, \\ Porto Alegre, RS, Brasil
}

\begin{abstract}
Resumo: O objetivo deste estudo foi verificar a influência da fadiga na técnica de pedalada durante teste de ciclismo máximo até a exaustão. Oito ciclistas treinados realizaram um teste incremental máximo e um teste de carga constante até a exaustão com 48 horas de intervalo. As forças resultante (FR) e efetiva (FE) aplicadas no pedal foram calculadas para a determinação do índice de efetividade (IE). A FE apresentou aumento entre o início e fim do teste (de $125 \pm 16$ para $169 \pm 52 \mathrm{~N}, \mathrm{p}=0,03$ ), enquanto o IE e a FR não apresentaram alterações (de $0,55 \pm 0,10$ para $0,59 \pm 0,08, p=0,14$; de $230 \pm 33$ para $284 \pm 72 \mathrm{~N}$, $\mathrm{p}=0,08$, respectivamente). O tornozelo apresentou aumento na amplitude de movimento (de $19^{\circ} \pm 4,69$ para $28 \pm 7,29 \circ, p<0,01)$. Os resultados sugerem que, durante teste de carga constante até a exaustão, ciclistas treinados mantêm a técnica de pedalada e que esta pode ser uma estratégia para manter a potência.
\end{abstract}

Palavras-chave: Fadiga. Músculo esquelético. Força no pedal. Desempenho. Ciclismo.

\section{Analysis of pedaling technique during cycling until exhaustion}

\begin{abstract}
The aim of this study was to investigate the effects of fatigue on the pedaling technique during maximal cycling test until exhaustion. Eight well-trained cyclists completed an incremental test and a constant workload cycling test until exhaustion with 48 hours of rest. The resultant (FR) and effective (FE) forces were calculated to obtain the index of effectiveness (IE). FE increased from the start to the end of the test (from $125 \pm 16$ to $169 \pm 52 \mathrm{~N}, \mathrm{p}=0.03$ ), whereas no differences were observed for IE and FR (from $0.55 \pm 0.10$ to $0.59 \pm 0.08, p=0.14$; from $230 \pm 33$ to $284 \pm 72 \mathrm{~N}, \mathrm{p}=0.08$, respectively). Increases in ankle joint range of motion were observed (from $19^{\circ} \pm 4.69$ to $28 \pm 7.29^{\circ}, p<0.01$ ). Results suggest that during cycling until exhaustion well-trained cyclists are able to sustain the pedaling technique, which can be an important strategy in order to sustain the power output.
\end{abstract}

Keywords: Fatigue. Skeletal muscle. Pedal force. Performance. Cycling.

\section{Introdução}

As forças aplicadas nos pedais têm sido utilizadas para descrever a técnica de pedalada de ciclistas. A avaliação da técnica é feita a partir da análise da magnitude e da orientação das componentes da força aplicada no pedal (TOO, 1990). Uma parcela da força total aplicada no pedal (força resultante - FR) é transmitida perpendicularmente ao pedivela, denominada força efetiva (FE), gerando torque propulsor (COYLE et al., 1991; CANDOTTI et al., 2007).

A proposta de um índice capaz de quantificar o percentual de aproveitamento da força aplicada no pedal foi introduzida por Lafortune; Cavanagh (1983). O índice de efetividade (IE) é definido como a razão entre FE e FR, sendo possível observar sua aplicação para o desempenho assim como para o processo de aprendizagem motora do gesto da pedalada (SANDERSON; CAVANAGH, 1990). Além da orientação das forças aplicadas no pedal, a técnica de pedalada depende do comportamento dos ângulos das articulações do quadril, joelho, tornozelo e, principalmente, do ângulo do pedal (KAUTZ et al., 1991).

Alguns estudos envolvendo o ciclismo investigaram as alterações na técnica de pedalada decorrentes de mudanças na cadência (CANDOTTI et al., 2007; $\underline{\text { ROSSATO }}$ et al., 2008), na carga de trabalho (KAUTZ et al., 1991), na posição do selim (DIEFENTHAELER et al., 2008) e na potência produzida (PATTERSON; 
MORENO, 1990; CARPES et al., 2005). No entanto, poucos estudos consideraram os efeitos do processo de instalação da fadiga, definida como incapacidade funcional na manutenção de um nível esperado de força (FITTS, 1994), na força aplicada no pedal e na cinemática da articulação do tornozelo e do pedal (SANDERSON; BLACK, 2003).

Amoroso et al. (1993) mensuraram as componentes normal e tangencial da força aplicada no pedal de 11 ciclistas competitivos pedalando a $300 \mathrm{~W}$ até a exaustão. Os resultados indicaram aumento no pico máximo do componente normal e redução da componente tangencial, sem alterações significativas na FR e na FE ao longo do teste. Em contrapartida, Sanderson; Black (2003) avaliaram ciclistas pedalando a $80 \%$ da potência aeróbia máxima (intensidade correspondente ao consumo máximo de oxigênio) até a exaustão e observaram aumento da componente normal e na FE durante a fase de propulsão da pedalada $\left(0-180^{\circ}\right)$ do início para o fim do teste.

Recentemente, Dorel et al. (2009) avaliaram as alterações nas forças aplicadas no pedal e no IE de 10 ciclistas treinados. Os atletas pedalaram até a exaustão em carga constante e equivalente a $80 \%$ da potência aeróbia máxima. Segundo os autores, ocorreu aumento da FR e FE entre $30-$ $150^{\circ}$ do ciclo de pedalada no final do teste ( $85 \%$ do tempo total), mas não foram observadas mudanças no IE nesse mesmo setor do ciclo.

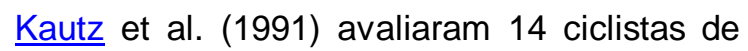
elite pedalando em duas cargas de trabalho e observaram padrões distintos para o ângulo do pedal. Parte dos atletas $(\mathrm{N}=7)$ avaliados apresentou aumento na amplitude de movimento (ADM) do pedal na medida em que aumentou a carga de trabalho, enquanto os demais não apresentaram mudanças. Os autores observaram aumento no pico de torque (que no ciclismo representa a relação entre $\mathrm{FE}$ e o tamanho do pedivela) no grupo de ciclistas que não apresentou alteração na ADM do pedal.

Na maioria das metodologias propostas para a análise da técnica da pedalada durante 0 processo de fadiga no ciclismo a carga do teste é submáxima (AMOROSO et al., 1993; SANDERSON; BLACK, 2003; DOREL et al., 2009), que para ciclistas de elite representa uma intensidade de esforço aquém do limiar anaeróbio. Dessa forma, descrever a técnica de pedalada em uma intensidade acima do limiar anaeróbio torna-se importante para identificar as estratégias neuromusculares utilizadas para minimizar os efeitos da fadiga. Estas estratégias são aplicadas não somente as provas de curta duração no ciclismo, mas também podem ser extrapoladas para provas de longa duração, como por exemplo, em situações com trechos em aclive quando o ciclista precisa otimizar sua técnica de pedalada para manter a potência.

Sendo assim, o objetivo do presente estudo foi verificar a influência da fadiga na técnica de pedalada de ciclistas treinados durante um teste de ciclismo máximo até a exaustão. A hipótese do presente estudo foi que à medida que ciclistas entram em processo de fadiga eles procuram melhorar o aproveitamento das forças aplicadas nos pedais de forma mais efetiva (ou seja, buscam melhorar o IE).

\section{Materiais e métodos}

\section{Sujeitos}

Oito ciclistas do sexo masculino foram selecionados para participar do presente estudo. Os sujeitos apresentavam em média 2 anos de experiência competitiva em provas nacionais (categoria 3 - USA Cycling Federation) e treinavam entre 350 e $600 \mathrm{~km} / \mathrm{semana}$. Durante o período da coleta de dados os ciclistas estavam se preparando para as seletivas nacionais da prova de $40 \mathrm{~km}$ contrarrelógio. Todos os procedimentos do estudo foram aprovados pelo comitê de ética em pesquisa com seres humanos da Universidade do Texas em Austin de acordo com as recomendações de Helsinki. Os sujeitos assinaram um termo de consentimento informado e evitaram realizar exercícios de alta intensidade ou exaustivos nas 24 horas precedentes aos testes.

\section{Procedimentos experimentais}

Os testes foram realizados em dois dias com 48 horas de intervalo. No primeiro dia foi realizado um teste submáximo com incremento de carga a cada três minutos $(50,60,70,80$ e $90 \%$ da frequência cardíaca máxima estimada (TANAKA et al., 2001), utilizando um cicloergômetro Lode Excalibur Sport V2.0 (Groningen, Holanda). Este modelo permite ajustes na altura e na posição do selim e do 
guidão, 0 que possibilita reproduzir 0 posicionamento do ciclista na sua própria bicicleta.

Ao longo da avaliação foram mensurados 0 consumo de oxigênio $\left(\mathrm{VO}_{2}\right)$, por meio de ergoespirometria de circuito aberto, usando um analisador de gases modelo Physiodyne FLO-1B System (Physio-Dyne Instrument Corp., New York, USA), e a frequência cardíaca (FC) usando um frequencímetro Polar S610 (Polar Electro Oy, Finlândia). Após esta etapa, enquanto os resultados da avaliação acima foram plotados em um gráfico da $\mathrm{FC}$ em função do $\mathrm{VO}_{2}$, e extrapolados para que fosse possível estimar 0 valor da potência mínima que deveria ser atingida durante o teste de consumo máximo de oxigênio $\left(\mathrm{VO}_{2 \mathrm{MAX}}\right)$, houve um período de recuperação de 15 minutos.

Para a determinação do $\mathrm{VO}_{2 \mathrm{MAX}}$ a carga inicial do teste foi correspondente a $60 \%$ do valor da potência mínima estimada $\left(\mathrm{W}_{\mathrm{MIN}}\right)$. Este estágio teve duração de quatro minutos, seguido de incrementos de carga a cada dois minutos $(75,90$ e $100 \%$ da $W_{\text {MIN }}$, respectivamente). Caso 0 ciclista não atingisse um platô dentro da carga estimada, incrementos de $5 \%$, a cada minuto, foram aplicados até a exaustão voluntária (ou interrompido quando o atleta não conseguisse manter a cadência acima de 70 rpm).

No segundo dia, os ciclistas realizaram um aquecimento durante 15 minutos a uma carga correspondente a $50 \%$ do $\mathrm{VO}_{2 \mathrm{MAX}}$, seguido de um protocolo máximo até a exaustão voluntária com carga referente a $100 \%$ do $\mathrm{VO}_{2 \mathrm{MAX}}$. A cadência de pedalada durante os testes foi a preferida do atleta e mantida constante ao longo do teste. Todos os ciclistas foram encorajados verbalmente durante o teste que era interrompido quando o atleta não conseguia manter a cadência de pedalada acima de $70 \mathrm{rpm}$ por um período superior a 5 segundos ou quando percebia que não poderia mais continuar. Durante o segundo dia de avaliação, paralelo ao $\mathrm{VO}_{2}$ e a $\mathrm{FC}$ foram mensuradas as forças aplicadas no pedal ao longo de todo o teste.

\section{Forças no pedal}

Para a mensuração da força muscular dos ciclistas foi utilizado um pedal-plataforma de força bidimensional instrumentado acoplado ao pedivela direito (NEWMILLER et al., 1988). Foram mensuradas simultaneamente as duas componentes da força aplicada no plano sagital, nas direções normal e tangencial, ao longo dos $360^{\circ}$ da pedalada. Os sinais de força foram registrados com o auxílio de um amplificador de sinais modelo 2021B (Vishay Measurements Group, EUA) e convertidos por uma placa analógico-digital de 64 canais e 32 bits de resolução do sistema Vicon 612 (Oxford Metrics, Inglaterra). Os dados de força obtidos do pedal instrumentado foram adquiridos a uma frequência de $1080 \mathrm{~Hz}$ de forma sincronizada aos dados de cinemetria. Os dados de força foram submetidos a um filtro digital Butterworth passa-baixa de $3^{\text {a }}$ ordem com frequência de corte de $10 \mathrm{~Hz}$ (MARSH et al., 2000), e foram então subamostrados para posterior sincronização com os dados de cinemetria.

A FR foi calculada considerando a soma vetorial das componentes normal e tangencial. Para o cálculo da FE foram somadas as componentes perpendiculares da força normal e tangencial (COYLE et al., 1991). A potência foi calculada a partir das informações da FE, da velocidade angular e do tamanho do pedivela. $O$ impulso angular da FE e o impulso linear da FR foram calculados para cada ciclo de pedalada para obtenção do IE (DIEFENTHAELER et al., 2008; BINI; DIEFENTHAELER, 2010). Posteriormente, o valor médio da FE e da FR, referente a 15 ciclos contínuos de pedalada, foi calculado para o período inicial, médio e final do teste máximo de ciclismo até a exaustão para cada sujeito. A análise dos dados foi conduzida utilizando rotinas desenvolvidas em ambiente Matlab $^{\circledR}$ (Mathworks Inc., EUA).

\section{Cinemetria}

Para calcular os ângulos da articulação do tornozelo, do pedivela, do pedal e do pedal e em relação ao pedivela foi utilizado um sistema de videografia tridimensional composto por seis câmeras infravermelho Vicon (Oxford Metrics, Inglaterra) e frequência de amostragem de 120 $\mathrm{Hz}$. As câmeras foram posicionadas ao redor da bicicleta (visão anterior, visão lateral e visão posterior, bilateralmente), permitindo assim a aquisição de imagens para a obtenção de ângulos articulares em qualquer um dos planos de movimento. As informações obtidas das seis 
câmeras foram digitalizadas instantaneamente por uma placa analógico-digital de 64 canais e 32 bits de resolução do sistema Vicon 612 (Oxford Metrics, Inglaterra). Marcadores reflexivos foram fixados no epicôndilo lateral do fêmur e no maléolo lateral do membro inferior direito, no eixo do pedal e nas extremidades anterior e proximal do pedal instrumentado.

A obtenção das coordenadas tridimensionais do movimento foi realizada por meio do método da transformação linear direta (DLT), utilizandose o software Vicon WorkStation (Oxford Metrics, Inglaterra). Esse método permite a reconstrução do movimento em três eixos, $z$ (médio-lateral), $x$ (ântero-posterior) e y (vertical) a partir das coordenadas obtidas no mínimo por duas câmeras. Destas, foram utilizadas no presente estudo apenas as variáveis nos eixos y e $\mathrm{x}$ para a análise bidimensional do movimento no plano sagital. Os dados de cinemática foram então submetidos a um filtro digital passa baixa do tipo Butterworth de $4^{\mathrm{a}}$ ordem, com frequência de corte de $15 \mathrm{~Hz}$. Posteriormente, foram calculados os ângulos do pedal relativo ao ângulo do pedivela, assim como os ângulos do pedal e do pedivela de forma independente (DIEFENTHAELER et al., 2008). A partir da posição vertical do pedal, foram calculados os intervalos de tempo relativos à maior posição do pedal no eixo vertical para a determinação da cadência de pedalada. Estas análises também foram realizadas utilizando rotinas matemáticas desenvolvidas em ambiente Matlab $^{\circledR}$.

\section{Análise estatística}

A normalidade da distribuição e a esfericidade dos dados foram verificadas por meio dos testes de Shapiro-Wilk e Mauchly, respectivamente. Os resultados do valor médio dos 15 ciclos de pedalada para a $\mathrm{FE}, \mathrm{FR}$, IE, $\mathrm{VO}_{2 \mathrm{MAX}}$, potência, cadência, ângulo e ADM do pedal e da articulação do tornozelo foram organizados para média e desvio-padrão do grupo de ciclistas com fins de posterior apresentação dos mesmos. A avaliação do processo de instalação da fadiga sobre a força aplicada nos pedais foi feita utilizando a análise de variância para medidas repetidas de um fator. Quando diferenças foram observadas, o teste Post Hoc LSD foi aplicado seguido de fator de correção de Holms para determinar quais níveis de comparação (início, meio ou fim do teste) apresentaram diferença significativa. Todas as análises estatísticas foram realizadas utilizando pacote estatístico SPSS 12.0 com nível de significância assumido para $\mathrm{p}<0,05$.

\section{Resultados}

Os resultados individuais e a média e desviopadrão (DP) do grupo para os dados de idade, massa corporal, consumo máximo de oxigênio $\left(\mathrm{VO}_{2 \mathrm{MAX}}\right)$, potência máxima (PO), frequência cardíaca máxima $\left(\mathrm{FC}_{\mathrm{MAX}}\right)$ e tempo para fadiga estão apresentados na Tabela 1.

Tabela 1. Características (média e DP) da idade, massa corporal, consumo máximo de oxigênio $\left(\mathrm{VO}_{2 \mathrm{MAx}}\right)$, potência máxima ( $\mathrm{PO}$ em Watts), frequência cardíaca máxima ( $\mathrm{FC}_{\mathrm{MAX}} \mathrm{em}$ bpm) e tempo de exaustão (TE em segundos) dos ciclistas avaliados.

\begin{tabular}{ccccccc}
\hline Sujeito & $\begin{array}{c}\text { Idade } \\
(\text { anos })\end{array}$ & $\begin{array}{c}\text { Massa Corporal } \\
(\mathrm{kg})\end{array}$ & $\begin{array}{c}\mathrm{VO}_{2 \mathrm{MAX}} \\
\left(\mathrm{ml} \cdot \mathrm{kg}^{-1} \cdot \mathrm{min}^{-1}\right)\end{array}$ & $\begin{array}{c}\mathrm{PO} \\
(\text { Watts })\end{array}$ & $\begin{array}{c}\mathrm{FC}_{\text {MAX }} \\
(\mathrm{bpm})\end{array}$ & $\begin{array}{c}\mathrm{TE} \\
(\mathrm{s})\end{array}$ \\
\hline S1 & 35 & 82,7 & 58,27 & 450 & 176 & 368 \\
S2 & 25 & 74,2 & 64,10 & 410 & 190 & 435 \\
S3 & 41 & 70,5 & 55,47 & 360 & 181 & 486 \\
S4 & 32 & 83,6 & 63,47 & 480 & 172 & 485 \\
S5 & 25 & 71,1 & 63,02 & 430 & 189 & 330 \\
S6 & 35 & 70,1 & 59,06 & 400 & 179 & 308 \\
S7 & 34 & 73,2 & 57,24 & 360 & 185 & 572 \\
S8 & 26 & 62,3 & 67,09 & 380 & 190 & 323 \\
\hline Média & 30,6 & 73,5 & 61,0 & 408 & 183 & 408 \\
DP & 5,80 & 6,96 & 4,01 & 40,24 & 6,84 & 91,35 \\
\hline
\end{tabular}


Os resultados da FE, FR, potência produzida, $\mathrm{VO}_{2}$, IE e cadência de pedalada dos ciclistas avaliados são apresentados na Figura 1.

Os ciclistas avaliados apresentaram um aumento da FE (de $125 \pm 16$ para $169 \pm 52 \mathrm{~N}$, respectivamente, $F_{1,096,7,673}=6,314, p=0,03$ ) entre o início e o fim do teste, mas não foram encontradas diferenças para FR (de $230 \pm 33$ para $284 \pm 72 \mathrm{~N}$, respectivamente, $F_{1,104,7,731}=3.832, p=0,08$ ), IE (de 0,55 $\pm 0,10$ para $0,59 \pm 0,08$, respectivamente, $F_{2,14}=2,215$, $p=0,14$ ) e potência (de $328 \pm 36$ para $302 \pm 34 \mathrm{~W}$,
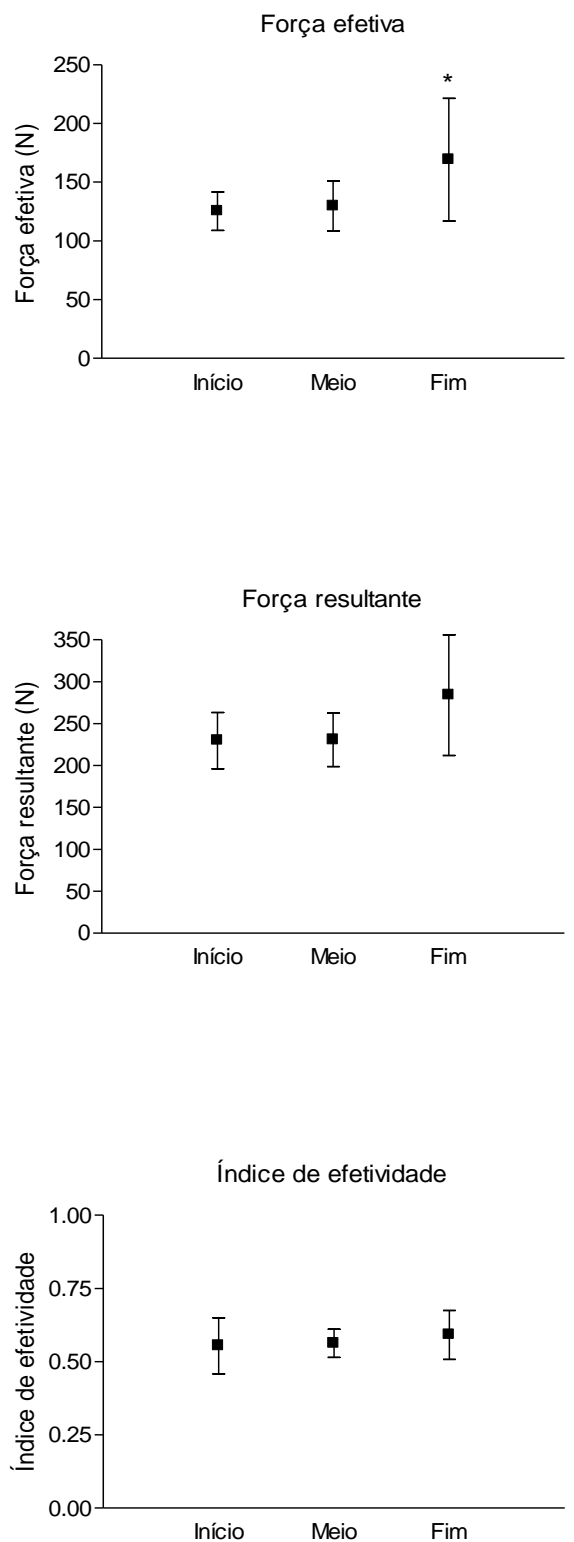

respectivamente, $F_{2,14}=2,492, p=0,69$ ). Para a cadência de pedalada foi observada uma redução significativa (de $94 \pm 5$ para $73 \pm 16 \mathrm{rpm}$, respectivamente, $\left.\quad \mathrm{F}_{2,14}=7,792, \quad \mathrm{p}<0,01\right)$ comparando o início e o fim do teste. $\mathrm{O} \mathrm{VO}_{2}$ apresentou aumento significativo ao longo do teste (de $50,5 \pm 6,64$ para $57,8 \pm 6,12 \mathrm{ml} \cdot \mathrm{kg}^{-1} \cdot \mathrm{min}^{-}$ ${ }^{1}$, respectivamente, $F_{2,14}=10,797, p<0,01$ ).

Os resultados dos ângulos médios do pedal e da articulação do tornozelo e as ADM do pedal e da articulação do tornozelo são apresentados na Tabela 2.
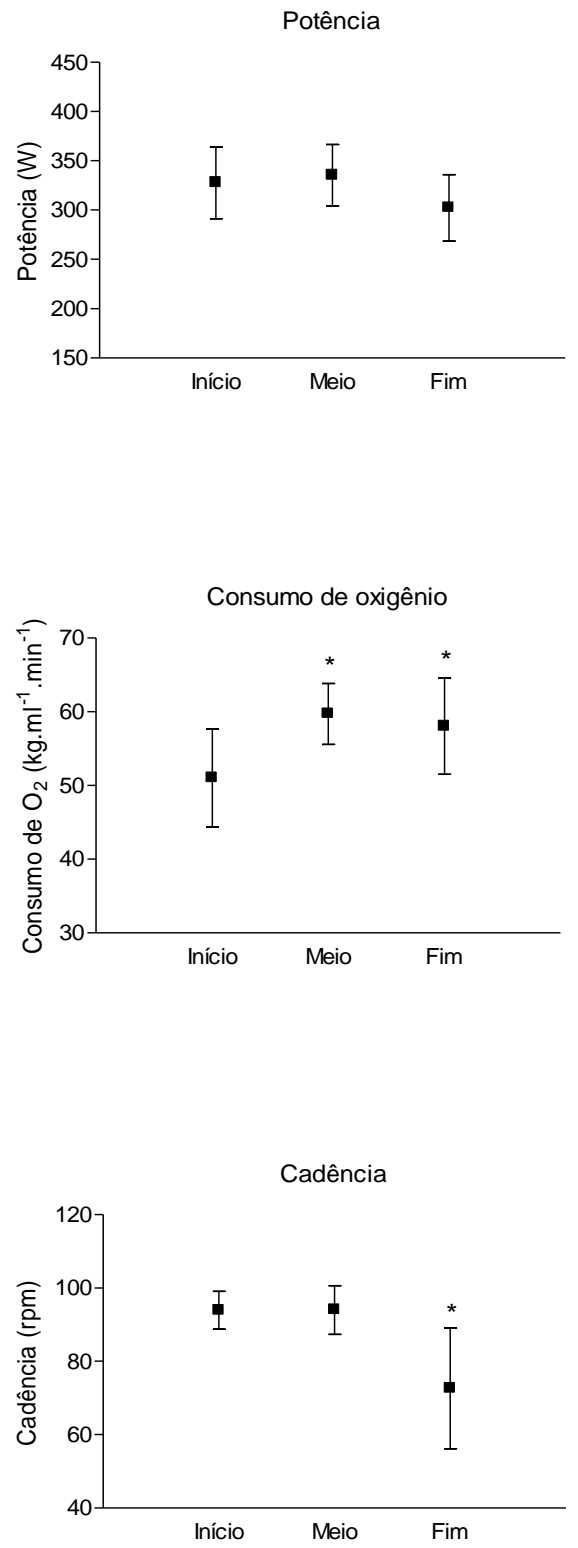

Figura 1. Médias e os desvios-padrão da força efetiva, força resultante, índice de efetividade, potência, consumo de oxigênio e cadência nos três instantes (início, meio e fim) do teste de carga constante $(\mathrm{N}=8)$. *diferença significativa em relação ao início do teste $(p<0,05)$. 
Tabela 2. Média e desvio-padrão do ângulo médio da articulação do tornozelo e do pedal e amplitude de movimento (ADM) do ângulo da articulação do tornozelo e do ângulo do pedal dos ciclistas avaliados $(\mathrm{N}=8)$.

\begin{tabular}{lcccccc}
\hline & \multicolumn{3}{c}{ Ângulo médio (graus) } & \multicolumn{3}{c}{ ADM (graus) } \\
\hline & Início & Meio & Fim & Início & Meio & Fim \\
Tornozelo & $157 \pm 5,52$ & $156 \pm 5,26^{\$}$ & $152 \pm 6,60^{\star \#}$ & $19 \pm 4,69$ & $20 \pm 6,70$ & $28 \pm 7,29^{\star \#}$ \\
Pedal & $6 \pm 10,17$ & $8 \pm 9,79$ & $11 \pm 6,52^{\star \#}$ & $69 \pm 7,05$ & $70 \pm 7,39$ & $78 \pm 7,7^{\star \#}$ \\
\hline
\end{tabular}

*diferença significativa entre início e fim $(p<0,01$ para ângulo médio do tornozelo, $p<0,01$ para ADM do ângulo médio do tornozelo, $p=0,04$ para o ângulo médio do pedal e $p<0,01$ para a ADM do ângulo do pedal). ${ }^{\$}$ diferença significativa entre início e meio do teste de fadiga ( $p=0,03$ e $p=0,01$ para o ângulo do tornozelo). "diferença significativa entre meio e fim do teste de fadiga ( $p<0,01$ para ângulo médio do tornozelo e ADM do ângulo médio do tornozelo, $p=0,03$ para o ângulo médio do pedal e $p<0,01$ para a ADM do ângulo médio do pedal).

Uma redução no ângulo médio da articulação do tornozelo foi observada ao longo do ciclo da pedalada do início para o meio do teste $(p=0,03)$, do início para o fim $(p<0,01)$ e do meio para o fim $(p<0,01)\left(F_{2,14}=32,942 ; p=0,03\right)$. A ADM do pedal também apresentou diferença significativa $\left(F_{2,14}=23,496 ; \quad p<0,01\right)$, aumentando do início para o fim do teste $(p<0,01)$ e do meio para o fim do teste $(p<0,01)$. Também foram observados aumentos no ângulo médio do pedal do início para o meio $(p=0,03)$ e do início para $\circ$ fim $(p=0,04)$ do teste e na ADM do pedal do início para o fim $(p<0,01)$ e do meio para o fim $(p<0,01)$ ao longo do teste de fadiga $\left(F_{2,14}=5,065 ; p<0,01\right.$; $F_{2,14}=14,250, p=0,02$, respectivamente).

\section{Discussão}

Este estudo se propôs a analisar o comportamento da técnica de pedalada de ciclistas ao longo de um teste de ciclismo realizado com carga máxima e constante até a exaustão. Três instantes (início, meio e fim) do teste foram analisados com o objetivo de verificar se o processo de instalação da fadiga poderia afetar o IE (por meio da análise do padrão de aplicação de força no pedal). Os ciclistas apresentaram aumento na FE no final do teste, mas não foram encontradas mudanças na $F R$ e IE. A partir destes resultados é possível sugerir que os ciclistas apresentaram melhor aproveitamento da força total aplicada no pedal ao longo do teste, confirmando parcialmente nossa hipótese inicial.

As consequências do processo de instalação da fadiga são apontadas como responsáveis pela redução na capacidade de produção de força muscular, o que repercute diretamente no desempenho de ciclistas (ST CLAIR GIBSON et al., 2001). Amoroso et al. (1993) observaram aumento no pico da força normal e redução na força tangencial de ciclistas treinados como consequência do processo de instalação da fadiga. No entanto, os ciclistas avaliados foram capazes de manter o mesmo padrão de FE, FR e IE ao longo de um teste com carga constante de $300 \mathrm{~W}$.

Em um estudo similar Sanderson; Black (2003) observaram que ciclistas competitivos não apresentaram alterações no IE do início para o fim de um teste de fadiga a $80 \%$ da potência aeróbia máxima. Segundo os autores, a manutenção do IE deve-se ao fato de que os ciclistas apresentaram aumento da FE durante a fase de propulsão com concomitante redução da FE durante a fase de recuperação $\left(180-360^{\circ}\right)$ ao longo do teste. O mesmo padrão na manutenção da técnica de pedalada foi observado por Dorel et al. (2009) quando avaliaram as forças no pedal de ciclistas treinados durante um protocolo de fadiga. Foi observado aumento da FR e FE em parte da fase de propulsão $\left(30-150^{\circ}\right)$, mas não foram observadas mudanças no IE nessa mesma parte do ciclo. Nossos resultados para FE e IE corroboram com os achados dos estudos de Sanderson; Black (2003) e Dorel et al. (2009).

No entanto, mesmo obtendo maior aproveitamento da força total aplicada no pedal no final do teste, os ciclistas avaliados no presente estudo não apresentaram melhora no IE. Lembrando que houve um aumento significativo na FE e que o IE é a razão entre a FE e a FR. Uma possível explicação para a manutenção do IE estaria no fato de que mesmo não sendo significativo ocorreu aumento da FR do meio para o fim do teste. Segundo Bini; Diefenthaeler (2010), ciclistas quando submetidos a condições máximas de carga de trabalho, apresentam alterações nos momentos articulares e ângulos articulares, sem alterar o IE. 
Segundo De Groot et al. (1994), a FE não depende somente da magnitude mas também da direção das forças, que por sua vez depende do ângulo do pedal (ângulo formado entre o pedal e a linha horizontal). Kautz et al. (1991) sugerem que o aumento da carga de trabalho resulta em um aumento da ADM do ângulo do pedal. De acordo os autores, essa estratégia permite ao ciclista aplicar força no pedal em ângulos mais favoráveis. Acreditamos que os ciclistas avaliados no presente estudo utilizaram a mesma estratégia, pois foi observado aumento na ADM do ângulo do pedal (Tabela 2) com concomitante aumento na FE (Figura 1) do início para o fim do teste.

Isso sugere ainda que as variações observadas no ângulo e na ADM do tornozelo podem indicar mudanças na propriedade forçacomprimento (FRASSON et al., 2007) e, consequentemente, alterar a capacidade de produção de força muscular do ciclista (HERZOG et al., 1991). Mais especificamente em relação aos flexores plantares do tornozelo, sabe-se que os mesmos atuam na fase ascendente da relação torque-ângulo (FRASSON et al., 2007), o que significa que esse grupo muscular aumenta a capacidade de produção de força com o aumento do comprimento muscular ou com o aumento do ângulo de flexão dorsal do tornozelo. Portanto, o aumento do ângulo de flexão dorsal da articulação do tornozelo com a fadiga sugere que os ciclistas aumentam o comprimento dos flexores plantares com o objetivo de se contrapor à redução de força decorrente da fadiga por meio de um aumento da capacidade de produção de força intrínseca dessa musculatura.

Dados de um estudo prévio (BINI et al., 2010) indicam aumento da FR nas três articulações analisadas, assim como alterações na cinemática das mesmas ao longo do teste (redução do ângulo médio da articulação do tornozelo, com aumento da ADM de flexão dorsal, aumento significativo do ângulo médio das articulações do joelho e do quadril). Os autores acreditam que a redução observada no ângulo médio da articulação do tornozelo (ou o aumento na dorsiflexão dessa articulação) é uma tentativa de aumentar a rigidez no tornozelo (por meio do aumento das forças ativa e passiva dessa musculatura) a fim de permitir a maior efetividade na transferência da força para 0 pedivela. Amoroso et al. (1993) observaram alterações nos ângulos mínimo e máximo da articulação do tornozelo do início para o fim em um teste de fadiga (de $108^{\circ}$ para $112^{\circ}$ no início da fase de propulsão, e de $85^{\circ}$ para $90^{\circ}$ no final da fase de propulsão, respectivamente). De acordo com os autores, o aumento da dorsiflexão altera o ângulo do pedal permitindo ao ciclista "puxar" o pedal para cima e para trás com mais eficiência durante a fase de recuperação. Dessa forma, o aumento observado na FE, no ângulo do pedal e na ADM do pedal e na articulação do tornozelo indica que os ciclistas avaliados no presente estudo utilizaram a estratégia da dorsiflexão para manter a potência constante até a exaustão.

Os resultados do presente estudo são contrários à expectativa de que o processo de instalação da fadiga resultaria em redução na capacidade de produção de força (NOAKES et al., 2004). Possivelmente o aumento nas forças pode estar associado às mudanças na resistência de frenagem do ciclo ergômetro, quando programado para operar em carga constante, de acordo com alterações na cadência de pedalada (MACINTOSH et al., 2000). O aumento das forças seria explicado por uma estratégia muscular na tentativa de manter a potência constante, pois em baixas velocidades de encurtamento os músculos são capazes de gerar mais força (ㅂILL, 1938).

Uma vez que a potência na pedalada depende da FE e da velocidade angular (cadência) e que os ciclistas avaliados no presente estudo apresentaram redução da cadência e aumento da FE do meio para o fim do teste, sugerimos que os ciclistas, para manter a potência constante até a exaustão, utilizaram a estratégia de reduzir a cadência de pedalada para aumentar a capacidade de gerar força no pedal (MACINTOSH et al., 2000). Se a redução da cadência de pedalada por um lado é decorrente dos efeitos deletérios da fadiga periférica sobre as fibras musculares, por outro visa otimizar a capacidade de produção de força das fibras musculares ainda não fatigadas por meio do aumento de sua capacidade de geração de força em menores velocidades angulares de movimento.

O aumento da FE observado no final do teste também pode estar relacionado ao fato de que em menores cadências de pedalada os ciclistas 
são capazes de "puxar" o pedal durante a fase de recuperação, melhorando dessa forma 0 aproveitamento das forças aplicadas no pedal (CANDOTTI et al., 2007). Segundo Sanderson et al. (2000), pedalar em baixas cadências permite ao ciclista aumentar a efetividade da pedalada durante a fase de recuperação minimizando assim o torque negativo gerado. Este comportamento indica que durante o processo de fadiga no ciclismo existe incapacidade de manutenção da cadência de pedalada para a mesma potência produzida (LEPERS et al., 2000).

Tem sido reportado que ciclistas optam por cadências de pedalada mais altas na tentativa de minimizar a fadiga periférica (TAKAISHI et al., 1998), reduzir a sobrecarga no sistema muscular (MARSH et al., 2000) e os momentos articulares resultantes (MORNIEUX et al., 2007). No entanto, essa estratégia em termos de otimização da técnica de pedalada parece não proporcionar melhoras, pois tem sido reportado que ciclistas pedalando acima da cadência preferida apresentam redução na FE (PATTERSON; MORENO, 1990; CANDOTTI et al., 2007). Como as forças no pedal são resultantes da ação muscular, uma redução da FE significaria um ineficiente trabalho muscular e, consequentemente, um desperdício de energia (ERICSON et al., 1988).

O consumo de oxigênio tem sido utilizado para medir a energia despendida e calcular a eficiência muscular no ciclismo (COYLE et al., 1992; MOSELEY; JEUKENDRUP, 2001). Os resultados do $\mathrm{VO}_{2 \mathrm{MAX}}$ e potência máxima dos ciclistas avaliados são compatíveis com o nível dos atletas (atletas experientes), uma vez que apresentaram valores entre 55 e $67 \mathrm{ml} \cdot \mathrm{kg}^{-1} \cdot \mathrm{min}^{-1}$, e 350 e $450 \mathrm{~W}$, respectivamente (MOSELEY; JEUKENDRUP, 2001). Estudos envolvendo ciclistas treinados utilizaram protocolos com carga constante para avaliar os efeitos do $\mathrm{VO}_{2}$ durante o processo de fadiga (LAJOIE et al., 2000; LEPERS et al., 2000; LUCIA et al., 2000). Os resultados desses estudos sugerem aumento no $\mathrm{VO}_{2}$ devido ao processo de instalação da fadiga, o que corrobora com os achados no presente estudo. $\mathrm{O}$ aumento no $\mathrm{VO}_{2}$ para uma mesma carga de trabalho vem sendo associado ao recrutamento de unidades motoras de maior calibre na tentativa da manutenção da potência desejada (LUCIA et al., 2000; MACDONALD et al., 2008). Além disso, o fato do teste de carga constante ter sido realizado no domínio severo ( $100 \%$ da potência máxima) explicaria o aumento observado no $\mathrm{VO}_{2}$, de maneira monoexponencial, até os seus valores máximos (GAESSER; POOLE, 1996).

A variabilidade aumentada para as variáveis analisadas no final do teste de carga constante indica que parece não ocorrer uma uniformidade na resposta mecânica decorrente do processo de instalação da fadiga no ciclismo. Alguns ciclistas podem apresentar comportamento distinto, o que poderia estar associado com adaptações específicas do treinamento, com características individuais do sistema músculo esquelético (i.e. distribuição do tipo de fibras musculares nos músculos relacionados), ou mesmo com aspectos técnicos (i.e. diferenças na estratégia técnica de aplicação de força).

\section{Conclusão}

Este estudo sugere que, sob condições de fadiga, ciclistas treinados são capazes de manter a técnica de pedalada (índice de efetividade) até a exaustão durante teste máximo de ciclismo. Pedalar em cadências mais baixas durante 0 processo de instalação da fadiga permite aplicar as forças de forma efetiva no pedal, o que parece ser uma importante estratégia para sustentar a potência desejada durante exercícios de alta intensidade.

\section{Referências}

AMOROSO, A.; SANDERSON D.J.; HENNING, E.M. Kinematic and kinetic changes in cycling resulting from fatigue. In 14th INTERNATIONAL CONGRESS OF BIOMECHANICS. Proceedings of 14th International Congress of Biomechanics. Paris - France, 1993. 94-95 p.

BINI, R.R.; DIEFENTHAELER, F. Kinetics and kinematics analysis of incremental cycling to exhaustion. Sports Biomechanics, Abingdon, v. 9, p. 223-235, 2010. doi:

http://dx.doi.org/10.1080/14763141.2010.540672

BINI, R.R.; DIEFENTHAELER, F.; MOTA, C.B. Fatigue effects on the coordinative pattern during cycling: Kinetics and kinematics evaluation.

Journal of Electromyography and Kinesiology, Oxford, v.20, n.1, p.102-107, 2010. doi:

http://dx.doi.org/10.1016/j.jelekin.2008.10.003 
CANDOTTI, C.T.; RIBEIRO, J.; SOARES, D.P.; OLIVEIRA, A.R.; LOSS, J.F.; GUIMARÃES, A.C. Effective force and economy of triathletes and cyclists. Sports Biomechanics, Abingdon, v.6, n.1, p.31-43, 2007. doi: http://dx.doi.org/10.1080/14763140601058490

CARPES, F.P.; BINI, R.R.; NABINGER, E.; DIEFENTHAELER, F.; MOTA, C.B.; GUIMARÃES, A.C.S. Aplicação de força no pedal em prova de ciclismo $40 \mathrm{~km}$ contra-relógio simulada: estudo preliminar. Revista Brasileira de Educação Física e Esporte, São Paulo, v.19, n.2, p.105-113, 2005. Disponível em: $<$ http://www.revistasusp.sibi.usp.br/pdf/rbefe/v19n 2/v19n2a02.pdf>. Acesso em: 06 mar. 2011.

COYLE, E.F.; FELTNER, M.E.; KAUTZ, S.A.; HAMILTON, M.T.; MONTAIN, S.J.; BAYLOR, A.M.; ABRAHAM, L.D.; PETREK, G.W. Physiological and biomechanical factors associated with elite endurance cycling performance. Medicine and Science in Sports and Exercise, Champaign, v.23, n.1, p.93-107, 1991. Disponível em:

$<$ http://www.edb.utexas.edu/coyle/pdf\%20library/( 40)\%20Coyle,\%20Feltner\%20et\%20al,\%20Physi ological\%20and\%20biochemical\%20determinants \%20of\%20elite\%20endurance\%20cycling\%20perf ormance,\%20Med\%20and\%20Sci\%20in\%20Spor ts\%20and\%20Exercise,\%2023,\%2093107,\%201991.pdf>. Acesso em: 06 mar. 2011.

COYLE, E.F.; SIDOSSIS, L.S.; HOROWITZ, J.F.; BELTZ, J.D. Cycling efficiency is related to the percentage of type I muscle fibers. Medicine and Science in Sports and Exercise, Champaign, v.24, n.7, p.782-788. 1992. Disponível em: $<$ http://www.edb.utexas.edu/coyle/pdf\%20library// 46)\%20Coyle,\%20Sidossis,\%20Horowitz,\%20Belt z.\%20Cycling\%20efficiency\%20is\%20related\%20 to\%20the\%20percentage\%20of\%20type\%201\%20 muscle\%20fibers,\%20Med\%20and\%20Sci\%20in \%20Sports\%20and\%20Exercise,\%2024(7)\%2078 2-8,\%201992.pdf>. Acesso em: 06 mar. 2011.

DE GROOT, G.; WELBERGEN, E.; CLIJSEN, L.; CLARIJS, J.; CABRI, J.; ANTONIS, J. Power, muscular work, and external forces in cycling. Ergonomics, London, v.37, n.1, p.31-42, 1994. doi:

\section{http://dx.doi.org/10.1080/00140139408963620}

DIEFENTHAELER, F.; BINI, R.R.; NABINGER, E.; LAITANO, O.L.; CARPES, F.P.; MOTA, C.B.; GUIMARÃES, A.C.S. Proposta metodológica para a avaliação da técnica da pedalada de ciclistas: estudo de caso. Revista Brasileira de Medicina do Esporte, São Paulo, v.14, p.155158, 2008. doi: http://dx.doi.org/10.1590/S1517$\underline{86922008000200014}$
DOREL, S.; DROUET, J.M.; COUTURIER, A.; CHAMPOUX, Y.; HUG, F. Changes of pedaling technique and muscle coordination during an exhaustive exercise. Medicine and Science in Sports and Exercise, Champaign, v.41, n.6, p.1277-1286, 2009. doi: http://dx.doi.org/10.1249/MSS.0b013e31819825f8

ERICSON, M.O.; NISELL, R. Efficiency of pedal forces during ergometer cycling. International Journal of Sports Medicine, Stuttgart, v.9, n.2, p.118-122, 1988.

FITTS, R. H. Cellular mechanisms of muscle fatigue. Physiological Reviews, Bethesda, v.74, n.1, p.49-94, 1994. Disponível em: $<$ http://physrev.physiology.org/content/74/1/49.ab stract>. Acesso em: 06 mar. 2011.

FRASSON, V.B.; RASSIER, D.E.; HERZOG, W.; VAZ, M.A. Dorsiflexor and plantarflexor torqueangle and torque-velocity relationships of classical ballet dancers and volleyball players. Brazilian Journal of Biomechanics, São Paulo, v.8, n.14, p.31-36, 2007. Disponível em: $<$ http://citrus.uspnet.usp.br/biomecan/ojs/index.ph p/rbb/article/view/48>. Acesso em: 06 mar. 2011.

GAESSER, G.A., POOLE, D.C. The slow component of oxygen uptake kinetics in humans. Exercise and Sport Science Review, New York, v.24, p.35-71, 1996.

HERZOG, W.; GUIMARÃES, A.C.S.; ANTON, M.G.; CARTER-ERDMAN, K.A. Moment-length relations of rectus femoris muscles of speed skaters/cyclists and runners. Medicine and Science in Sports and Exercise, Champaign, v.23, n.11, p.1289-1296, 1991.

HILL, A.V. The Heat of Shortening and the Dynamic Constants of Muscle. Proceedings of the Royal Society of London. Series B, Biological Sciences, London, v.126, n.843, p.136-195, 1938. Disponível em: $<$ http://rspb.royalsocietypublishing.org/content/12 6/843/136.full.pdf>. Acesso em: 24 out. 2011.

KAUTZ, S. A., FELTNER, M.E.; COYLE, E.F.; BAYLOR, A.M. The pedaling technique of elite endurance cyclists: changes with increasing workload at constant cadence. International Journal of Sport Biomechanics, Champaign, v.7, n.1, p.29-53, 1991.

LAFORTUNE, M.A.; CAVANAGH P.R. Effectiveness and efficiency during bicycle riding. In: Matsui \& Kobashi K (Ed.). Biomechanics VIIIB: Human Kinetics, 1983, p.928-36.

LAJOIE, C.; LAURENCELLE, L.; TRUDEAU, F. Physiological responses to cycling for 60 minutes 
at maximal lactate steady state. Canadian Journal of Applied Physiology, Champaign, v.25, n.4, p.250-261, 2000. doi: http://dx.doi.org/10.1139/h00-019

LEPERS, R., HAUSSWIRTH, C.; MAFFIULETTI, N.; BRISSWALTER, J.; VAN HOECKE, J. Evidence of neuromuscular fatigue after prolonged cycling exercise. Medicine and Science in Sports and Exercise, Champaign, v.32, n.11, p.1880-1886, 2000. Disponível em: $<$ http://www.onlinetri.com/sites/romualdlepers/documents/LepersMSSE2000.pdf>. Acesso em: 06 mar. 2011.

LUCIA, A., HOYOS, J.; CHICHARRO, J.L. The slow component of $\mathrm{VO} 2$ in professional cyclists. British Journal of Sports Medicine,

Loughborough, v.34, n.5, p.367-374, 2000. doi: http://dx.doi.org/10.1136/bjsm.34.5.367

MACDONALD, J.H.; FARINA, D.; MARCORA, S.M. Response of electromyographic variables during incremental and fatiguing cycling. Medicine and Science in Sports and Exercise, Champaign, v.40, n.2, p.335-344, 2008. doi: http://dx.doi.org/10.1249/mss.0b013e31815b491e

MACINTOSH, B.R., NEPTUNE, R.R.; HORTON, J.F. Cadence, power, and muscle activation in cycle ergometry. Medicine and Science in Sports and Exercise, Champaign, v.32, n.7, p.1281-1287, 2000. Disponível em: <http://www.me.utexas.edu/ neptune/Papers/mss e32(7). $p d f>$ Acesso em: 06 mar. 2011.

MARSH, A.P.; MARTIN, P.E.; SANDERSON, D.J. Is a joint moment-based cost function associated with preferred cycling cadence? Journal of Biomechanics, Elmsford, v.33, n.2, p.173-180, 2000. Disponível em: $<$ http://educ.ubc.ca/faculty/sanderson/lab/CLFbio m/Downloads/Marsh,\%20Martin,\%20Sanderson.p df >. Acesso em: 06 mar. 2011.

MORNIEUX, G.; GUENETTE J.A.; SHEEL, A.W.; SANDERSON D.J. Influence of cadence, power output and hypoxia on the joint moment distribution during cycling. European Journal of Applied Physiology, Berlin, v.102, n.1, p.11-18, 2007. Disponível em:

$<$ http://www.springerlink.com/content/6236124448 2886ju/fulltext.pdf $>$. Acesso em: 27 jan. 2012.

MOSELEY, L.; JEUKENDRUP, A.E. The reliability of cycling efficiency. Medicine and Science in Sports and Exercise, Champaign, v.33, n.4, p.621-627, 2001. Disponível em: $<$ http://edulife.com.br/dados\%5CArtigos\%5CEduc acao\%20Fisica\%5CFisiologia\%20do\%20Exercici 0\%5CThe\%20reliability\%20of\%20cycling\%20effic iency.pdf>. Acesso em: 06 mar. 2011.
NEWMILLER, J.; HULL, M.L.; ZAJAC, F.E. A mechanically decoupled two force component bicycle pedal dynamometer. Journal of

Biomechanics, Elmsford, v.21, n.5, p.375-386, 1988. doi: http://dx.doi.org/10.1016/0021$\underline{9290(88) 90144-3}$

NOAKES, T.D.; ST CLAIR GIBSON, A. Logical limitations to the "catastrophe" models of fatigue during exercise in humans. British Journal of Sports Medicine, Loughborough, v.38, n.5, p.648-649. 2004. Disponível em:

$<$ http://www.ncbi.nlm.nih.gov/pmc/articles/PMC17 24943/pdf/v038p00648.pdf>. Acesso em: 06 mar. 2011.

PATTERSON, R.P.; MORENO, M.J. Bicycling pedaling forces as a function of pedaling rate and power output. Medicine and Science in Sports and Exercise, Champaign, v.22, n.4, p.512-516, 1990.

ROSSATO, M., BINI, R.R.; CARPES, F.P.; DIEFENTHAELER, F.; MORO, A.R. Cadence and workload effects on pedaling technique of welltrained cyclists. International Journal of Sports Medicine, Stuttgart, v.29, n.9, p.746-752. 2008. doi: http://dx.doi.org/10.1055/s-2008-1038375

SANDERSON, D.J.; BLACK, A. The effect of prolonged cycling on pedal forces. Journal of Sports Science, Bursa, v.21, n.3, p.191-199, 2003. doi:

http://dx.doi.org/10.1080/0264041031000071010

SANDERSON, D.J.; CAVANAGH, P.R. Use of augmented feedback for the modification of the pedaling mechanics of cyclists. Canadian Journal of Sport Sciences, Vanier City, v.15, n.1, p.38-42, 1990.

SANDERSON, D.J.; HENNIG, E.M.; BLACK, A.H. The influence of cadence and power output on force application and in-shoe pressure distribution during cycling by competitive and recreational cyclists. Journal of Sports Science, Bursa, v.18, n.3, Mar, p.173-181, 2000. Disponível em: $<$ http://educ.ubc.ca/faculty/sanderson/lab/CLFbio m/Downloads/Sanderson,\%20Hennig\%20and\%20 Black.pdf>. Acesso em: 06 mar. 2011.

ST CLAIR GIBSON, A.; SCHABORT, E.J.; NOAKES, T.D. Reduced neuromuscular activity and force generation during prolonged cycling.

American Journal of Physiology - Regulatory, Integrative and Comparative Physiology, Bethesda, v.281, n.1, p.R187-R196, 2001. Disponível em: $<$ http://ajpregu.physiology.org/content/281/1/R187 .full.pdf+html>. Acesso em: 06 mar. 2011. 
TAKAISHI, T., YAMAMOTO, T.; ONO, T.; ITO, T.; MORITANI, T. Neuromuscular, metabolic, and kinetic adaptations for skilled pedaling performance in cyclists. Medicine and Science in Sports and Exercise, Champaign, v.30, n.3, p.442-449, 1998. doi: http://dx.doi.org/10.1097/00005768-199803000$\underline{00016}$

TANAKA, H.; MONAHAN, K.D.; SEALS, D.R. Age-predicted maximal heart rate revisited. Journal of the American College of Cardiology, New York, v.37, p.153-156, 2001. Disponível em:

$<$ http://content.onlinejacc.org/cgi/reprint/37/1/153. pdf>. Acesso em: 27 jan. 2012.

TOO, D. Biomechanics of cycling and factors affecting performance. Sports Medicine, Auckland, v.10, n.5, p.286-302, 1990.

Agradecimentos: Os autores gostariam de agradecer ao CNPq e Capes pelo aporte financeiro para a realização deste projeto, aos professores Edward Coyle e Richard Neptune da Universidade do Texas em Austin (EUA), assim como todos os ciclistas que participaram deste projeto.

Endereço:

Fernando Diefenthaeler UFSC - Centro de Desportos - Campus

Universitário Trindade

Florianópolis SC Brasil

88040-970

Telefone: (48) 3721-8530

e-mail: fernando.diefenthaeler@ufsc.br

Recebido em: 17 de março de 2011.

Aceito em: 1 de fevereiro de 2012.

\section{(a) (1)}

Motriz. Revista de Educação Física. UNESP, Rio Claro, SP, Brasil - elSSN: 1980-6574 - está licenciada sob Creative

Commons - Atribuicão 3.0 